\title{
Microcellular Propagation Prediction Model Based on a Geometric Progression Approximation-Process
}

\author{
Antonis G. Dimitriou, Student Member, IEEE, and George D. Sergiadis, Member, IEEE
}

\begin{abstract}
A new propagation prediction model, suitable for an antenna placed below rooftop level in an urban environment is presented. It is shown that the contribution of various arriving rays at a specific location can be well approximated by a set of geometric progressions. Each progression is associated with a source. Then, estimation is delivered as soon as the leading term and the ratio have been computed. The ratio reflects the significance of secondary ray-paths. It depends on the geometry and the electromagnetic properties of the materials within the "street canyon." In contrast to typical semi-deterministic approaches, detailed information on the power, the time of arrival and the angle of arrival of all significant components is delivered. Consequently, wideband characterization of the environment is accomplished at short running-time. High accuracy is experimentally verified in a typical urban configuration.
\end{abstract}

Index Terms-Diffraction, propagation modeling, radiocoverage, ray tracing, uniform theory of diffraction (UTD), urban areas.

\section{INTRODUCTION}

A MONG the key problems in the design and planning phases of modern microcellular networks are accurate path loss prediction and proper characterization of the impact of scattering effects on the system's performance. Propagation prediction models are used to help designers. They can be distinguished in three main categories [1]-[3]: empirical, semi-deterministic and deterministic ones.

Empirical models [4] have a limited range of application, as they are based on measurements and not directly on the associated physical phenomena.

On the other hand, deterministic models, [5], [6], based on ray-theory and the uniform theory of diffraction (UTD), [7], [8], have been proven exceptionally accurate [9], at the expense of high-complexity and large running-time. A tradeoff between running-time and accuracy can be accomplished by implementing progressive strategies [10], [11].

In between, lay semi-deterministic models. Fast prediction is delivered after gathering some characteristics of the environment. This property places them on top of the list for radio planning, cell design, and system optimization.

Numerous models have been developed for the specific case of a transmitting antenna placed above or at the average rooftop

Manuscript received December 8, 2005; revised October 7, 2006

The authors are with the Aristotle University of Thessaloniki, School of Engineering, Department of Electrical \& Computer Engineering, Telecommunications Laboratory, Thessaloniki GR-54124, Greece (e-mail: antodimi@mri.ee. auth.gr; sergiadi@auth.gr).

Digital Object Identifier 10.1109/TAP.2007.891813 level [12]-[15]. Others have been introduced for an antenna placed below rooftop level, to account for the related propagation mechanisms [4], [16]-[18] (lateral instead of vertical propagation [13]). For the sake of simplicity and short running time, their philosophy focuses on identifying and calculating the contribution of a dominant path. However, thorough analysis and measurements have shown that the narrowband power level is determined by the superposition of several components in the "street canyon" [9], [19]. Therefore, so far, such approaches, [16], [17], are limited in a rough estimation of path loss and deliver no information on the wideband characteristics of the channel. In addition, in [17], a constant reflection coefficient is assumed throughout the area of interest, while in [16] the effects of different materials are not included in the calculations. Both assumptions may lead to large errors, as shown in [9].

We propose a new two dimensional propagation prediction model for an antenna placed below rooftop level that bridges the gap between deterministic and semi-deterministic approaches, and combines their advantages. High accuracy is accomplished by considering the buildings' arrangement in the area of interest, the location of the receiver therein, the electromagnetic (EM) properties of the materials involved, the different possible mechanisms that link the receiver with the transmitter (diffraction and multiple reflections) and the possible existence of foliage that may strongly influence the propagating EM wave. We shall show that the contribution of various arriving components at a specific location can be approximated by a set of geometric progressions with ratios $r_{i}<1$. Each progression is associated with a source; either main (transmitter) or secondary (through diffraction). Then, calculation of all propagation related characteristics can be accomplished by specifying the leading term of each sequence and the corresponding ratio. High accuracy and wideband characterization of the specific environment is accomplished in a short running-time (comparable to [16] and [17]). Compared to a deterministic ray-tracing model significant time is saved by avoiding the exhaustive search of all multiply reflected rays in the propagation area. Additionally, we show that the geometric progression's ratio associated with a specific location, depends on the geometry and the materials of the surrounding environment, and thus represents a new measure for the characterization of the scattering behavior of the environment.

The proposed formulation is introduced in Section II for a line of sight (LOS) case, where side streets are not considered. In Section III, the model is extended for a non line of sight (NLOS) situation. The effects of side streets on the propagating EM wave are analyzed and included in the proposed formulation in Section IV. The implementation of the model in a ray-tracing 
tool, experimental results and important properties are presented in Section V and finally we conclude in Section VI.

\section{LINE OF SIGHT ANALYSIS}

Low elevated antennas are typically used to serve the increased load that is generated at dense urban areas during the "busy hours." In such cases of high-traffic conditions, the ground reflected ray is obstructed by parked and moving vehicles, other "street furniture" (e.g., covered bus stops) and pedestrians. As a result it reaches the receiver greatly attenuated and can be excluded from calculations.

Let us consider a straight street of width $d$, with a transmitter placed on one side of it and the receiver at the center at a distance $R$ along the $x$-axis, as shown in Fig. 1. Both antennas are considered at the same height. Similarly to [16] and [17], we assume that the buildings' facades are flat, smooth and infinitely high, and the ground-reflected ray is neglected. Rays arrive at the receiver having traveled different paths, after being multiply reflected at both sides of the canyon. The phases of successive arriving components can be safely considered uncorrelated, as long as the difference between the distances traveled by any two rays is greater than the wavelength of operation $\lambda$. The range for which this assumption is valid depends on the width of the street and the distance from the source. It can be easily proven that the minimum difference is the one between the direct and the singly reflected ray. Hence

$$
\begin{aligned}
\lambda & \leq \min _{i, j=\{0,1, \ldots\}}\left|S_{i}-S_{j \neq i}\right| \\
& =d\left[\left[k^{2}+\left(\frac{3}{2}\right)^{2}\right]^{1 / 2}-\left[k^{2}+\left(\frac{1}{2}\right)^{2}\right]^{1 / 2}\right]
\end{aligned}
$$

where $S_{i}$ is the distance traveled by the $i$ th ray and $k=R / d$. For a typical $20-\mathrm{m}$ width street and $2 \mathrm{GHz}$ frequency of operation, phases remain uncorrelated for more than $2.5 \mathrm{~km}$. Besides, for such greater distances other wave-guiding phenomena, due to the facades of the surrounding buildings, ought to be included [20]. In such cases, the average power $\hat{P}$ (in Watts) at the receiver is proportional to the sum of the squares of the electric-field components $E_{i}$ (similar conclusion can be found in [21]). Therefore

$$
\hat{P}=\frac{\lambda^{2}}{4 \pi} \frac{1}{2 \eta} \sum_{i} G_{R}\left(\phi_{i}\right) E_{i}^{2}
$$

where $\eta$ is the free space impedance and $G_{R}\left(\phi_{i}\right)$ is the gain of component $i$, due to the receiving antenna. For longer distances, where (1) becomes invalid, the exact phase-sum should be calculated [21].

In (2), $E_{i}$ is proportional to the square root of the gain of the transmitting antenna $\sqrt{G_{T}\left(\phi_{i}{ }^{\prime}\right)}$. At greater distances, the angles of arrival $\varphi_{i}$ of the multiply reflected rays at the receiver do not differ significantly from that of the direct ray $\varphi_{0}$ (Fig. 1). Similarly, the angles of departure $\varphi_{i}^{\prime}$ of the above rays from the source are also close to that of the direct ray $\varphi_{0}^{\prime}$. Therefore, in most cases, the gains $G_{R}\left(\phi_{i}\right), G_{T}\left(\phi_{i}{ }^{\prime}\right)$ of the multiply reflected components may be substituted by those of the direct ray $G_{R d}, G_{T d}$ respectively. Without loss of generality, we assume

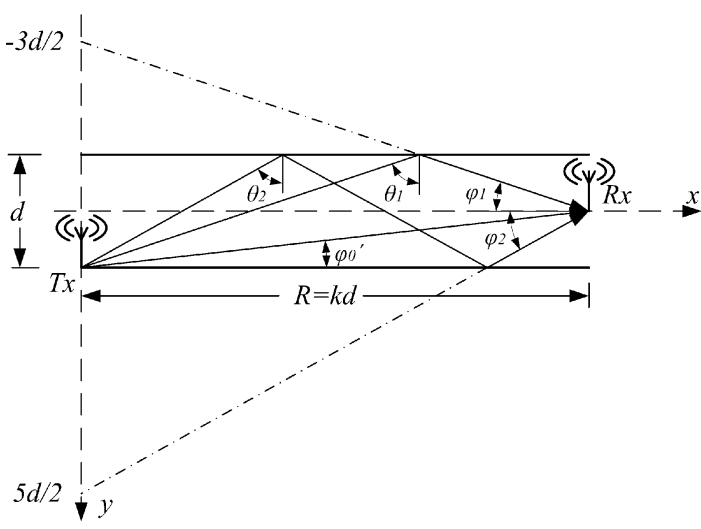

Fig. 1. Representation of the LOS configuration.

that the electric field is linearly polarized along a line perpendicular to the ground. After some simplifications, (2) can be written as

$$
\hat{P}=C \sum_{i} \frac{1}{S_{i}^{2}}\left[\left(\Gamma_{i}^{\perp}\right)^{2}\right]^{i}, \text { with } C=\left(\frac{\lambda}{4 \pi}\right)^{2} G_{R d} G_{T d} W_{T}
$$

where $W_{T}$ is the transmitted power (in Watt) and $\Gamma_{i}^{\perp}$ is the corresponding reflection coefficient for perpendicular polarization that depends only on the angle of incidence. In (3), for $i=0$, we calculate the contribution of the direct ray, while greater values of $i$ correspond the $i$ th reflected ray. Had the electric field component that is polarized parallel to the ground been considered, a similar series would have been constructed with $A^{\perp}$ and $A^{\| l}$ the constant terms and $\Gamma_{i}^{\|}$the corresponding reflection coefficient for the parallel polarization. In (3), each subsequent term has traveled a longer path $\left(S_{i}\right)$ and suffered an additional reflection, as depicted in the increasing exponent of the reflection coefficient. Equation (3) can be written as

$$
\hat{P}=\frac{C}{d^{2}} \sum_{i} \frac{1}{k^{2}+\left(\frac{(2 i+1)}{2}\right)^{2}}\left[\Gamma_{\perp}^{2}\left(\tan ^{-1}\left(\frac{2 k}{2 i+1}\right)\right)\right]^{i} .
$$

The ratio among any two successive terms $i$ and $i-1(i>0)$ is then given by

$$
r_{i}=\frac{\left[\Gamma_{\perp}^{2}\left(\tan ^{-1}\left(\frac{2 k}{2 i+1}\right)\right)\right]^{i}\left[k^{2}+\left(\frac{(2 i-1)}{2}\right)^{2}\right]}{\left[\Gamma_{\perp}^{2}\left(\tan ^{-1}\left(\frac{2 k}{2 i-1}\right)\right)\right]^{i-1}\left[k^{2}+\left(\frac{(2 i+1)}{2}\right)^{2}\right]} .
$$

The ratio among any two consecutive terms is not constant, but depends on the slope of the magnitude of the reflection coefficient and the distance depending term. No safe estimation of a single ratio $r$ can be deduced. However, in (4), we have succeeded to express the multiply reflected field at any point of a street, as the product of a simple term that depends on the width of the specific street and a sum that depends on the EM properties of the surrounding environment and on $k$. The sum depends on the position of the receiver and the street's width through $k=R / d$. For different streets with different widths $d_{i}$ but with identical materials, this sum reaches the same value at distances $R_{i}=k d_{i}$. This important property will be analyzed and exploited in the following paragraphs. 

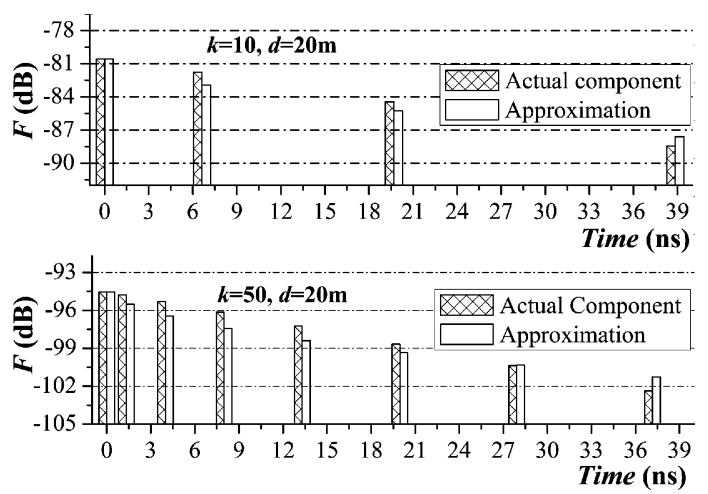

Fig. 2. Comparison between the exact and the estimated power profile of the arriving components with respect to the time of arrival of the direct term.

Finally, we wish to approximate (4) with a geometric series. We seek for a suitable ratio $r_{\mathrm{opt}}(k)$, so that the resulting sum is the same and the 1 st term is given by the power of the LOS ray $P_{\text {LOS: }}$

$$
\hat{P}=\frac{C}{d^{2}} \sum_{i} \frac{1}{k^{2}+\left(\frac{1}{2}\right)^{2}}\left(r_{\mathrm{opt}}(k)\right)^{i}=P_{\mathrm{LOS}} \frac{1}{1-r_{\mathrm{opt}}(k)}
$$

In (6), we have assumed 1) an infinite number of contributing terms and 2) that $r_{\mathrm{opt}}(k)<1$. The 1 st assumption is valid for the scenario of Fig. 1, where side streets are not taken into consideration. Side street contribution will be analyzed in Section IV. The validity of the 2nd assumption can be easily verified by inspecting (3), since each higher order term has traveled a greater path $S_{i}$ and suffered an additional reflection with magnitude $\Gamma_{i}^{\perp \|} \leq 1$.

As pointed out earlier, the sum in (4) depends only on the EM properties of the walls in the canyon. Hence, in a preprocessing phase, it could be easily calculated for several values of $k$. For these values an $r_{\text {opt }}(k)$ can be assigned by applying (6). Then, during the prediction process for random values of $k$, a linear interpolation is assumed for $r_{\mathrm{opt}}(k)$ and (6) is again invoked. As it will be shown in Section V, it is advantageous to calculate $r_{\mathrm{opt}}(k)$ since it carries information about the material of the walls and the position of the receiver with respect to the transmitter, therefore acting as a characteristic property of the propagation area. Additionally, the analysis so far will be proven useful also for the NLOS case.

A comparison between the exact and the approximated components for $d=20 \mathrm{~m}$ and $k_{1,2}=10,50$ is presented in Fig. 2 . $F$ is given by $F=10 \log _{10}\left(P_{R} / P_{T}\right)$. The time of arrival of different terms is measured with respect to the direct term. Only the components that changed the total received power by more than $0.1 \mathrm{~dB}$ are included in the graphs. Notice that the 1 st 4 terms dominate in the 1 st case, whereas a total of 8 terms have to be included in the 2nd case, because at greater distances the reflection coefficient of higher order terms is greater and the variation of the distance-depending term is smaller. This remark is particularly important, since it reveals that the number of important terms is directly associated with the materials and the geometry

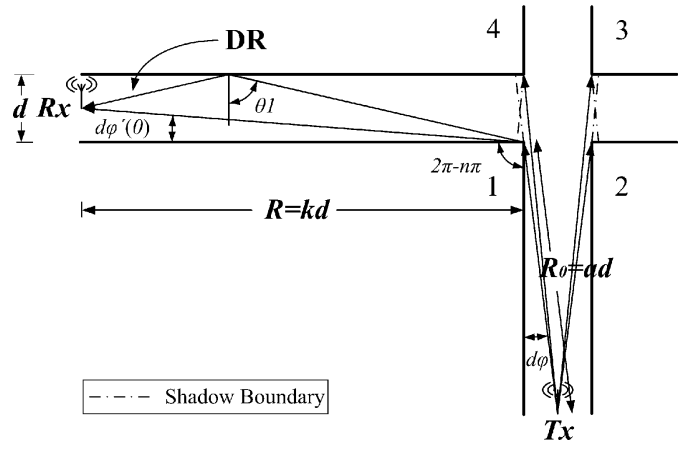

Fig. 3. Representation of the NLOS configuration.

of the environment and that no general rule can safely describe a specific environment [9].

The terms of the proposed geometric progression tend to slightly underestimate the contribution of the initial components (after the direct one), and to overestimate the latter, resulting in a balanced total contribution. This behavior was expected, since the geometric progression approximates the exact variation of the reflection coefficient in (4) (reflections of higher order occur at greater angles $\theta_{i}$, resulting in smaller magnitude). The average absolute difference between the actual component and the approximated one is kept small for both cases $(0.7 \mathrm{~dB})$. The estimation of the average received power is accurate, as defined by (6).

The presented description of the arriving rays carries additional information, compared to classic semi-deterministic pathloss only models, which is crucial for the analysis of the effects of multipath for several site-specific applications (antenna design, multiple input multiple output systems, modulation and coding evaluation etc.). The magnitude $P_{i}$, the time of arrival $t_{i}$ and the angle of arrival of successive components $\varphi_{i}$, are summarized in the following set of equations:

$$
\begin{aligned}
P_{i} & =P_{\mathrm{LOS}}\left(r_{\mathrm{opt}}\right)^{i}(\text { Watt }) \\
t_{i} & =\frac{d}{c}\left[k^{2}+\left(\frac{(2 i+1)}{2}\right)^{2}\right]^{1 / 2}(s), \quad i \geq 0 \\
\varphi_{i} & =\arctan \left((-1)^{i} \frac{(2 i+1)}{2 k}\right)
\end{aligned}
$$

where $c$ is the speed of light.

\section{NON LINE OF Sight ANALYSIS}

Let us consider the typical configuration, shown in Fig. 3 . Different rays reach the receiver either after being multiply reflected at the vertical walls of the canyon, or after being initially diffracted at the vertical walls of the cross junction and then multiply reflected at the facades of the side street (DR). The contribution of the latter becomes dominant after the immediate vicinity of the cross junction [1], [9], [18], [20]-[23]. In the following analysis only these rays will be considered. 
Adopting a similar approach with the one already presented for the LOS case the DR average power due to a wedge in the side street is given by

$$
\begin{aligned}
\hat{P} & =P_{W} \sum_{i} Y(i)\left[\left(\Gamma_{i}^{\perp}\right)^{2}\right]^{i}, \text { where } Y(i)=A(i)|D(i)|^{2} \\
\text { and } A(i) & =\frac{R_{0}}{S(i)\left(R_{0}+S(i)\right)} \\
\text { or } A(i) & =\frac{1}{d} \frac{a}{\left[k^{2}+\left(\frac{(2 i+1)}{2}\right)^{2}\right]^{1 / 2}\left(a+\left[k^{2}+\left(\frac{(2 i+1)}{2}\right)^{2}\right]^{1 / 2}\right)} .
\end{aligned}
$$

In (8), $P_{W}$ is the incident power at the vertical wedge, $A(i)$ is the distance depending term, $D(i)$ is the heuristic diffraction coefficient [8], corresponding to the $i$ th reflected ray, $R_{0}$ is the distance traveled from the source to the wedge, $S(i)$ is the distance traveled after the diffraction, from the wedge to the receiver, $k=R / d$ and $a=R_{0} / d$.

Compared with (4) a new variable $a$ has been added. Moreover, the diffraction coefficient $D(i)$ depends on the interior wedge angle and the material. If a similar procedure to that for the LOS case was followed, exhaustive preprocessing would be required to account for the variation of the above parameters. However, it is desirable to keep the complexity of the final approach low.

Let us consider the function $\Lambda(i)=Y(i+1) / Y(i), i \geq 0$. As demonstrated in Fig. 3, in all typical urban geometries, diffraction occurs away from the two shadow boundaries. The validity of this assumption grows at increasing distance from the cross junction. In the appendix, it is shown that when diffraction occurs away from the two shadow boundaries and the magnitude of the diffraction coefficient is different than 0 , then the derivative of $\Lambda(i)$ with respect to $i$ approaches 0

$$
\frac{d \Lambda(i)}{d i} \approx 0 \Leftrightarrow \Lambda(i)=r_{d} \Leftrightarrow \frac{A(i+1)|D(i+1)|^{2}}{A(i)|D(i)|^{2}}=r_{d}
$$

where $r_{d}$ is constant for each receiver's position. Hence $r_{d}$ can be specified by calculating $Y(0)$ (diffracted ray) and $Y(1)$ (diffracted-reflected ray) for each receiver's position.

Following a similar procedure to that of Section II, the reflection-coefficient terms in (8) can also be approximated with a suitable geometric progression in a preprocessing phase for several values of $k$. In such a case, the power of the $i$ th arriving ray will be given by

$$
P_{i}=P_{W} Y(0) r_{d}^{i} r_{R}^{i}=P_{\mathrm{SD}}\left(r_{d} r_{R}\right)^{i}=P_{\mathrm{SD}}\left(r_{\mathrm{opt}}\right)^{i}
$$

where $r_{d}$ and $r_{R}$ are the corresponding geometric-progressions' ratios and $P_{\mathrm{SD}}$ is the power of the singly diffracted ray. Notice the analogy between (10) and (7). In a similar approach, time of arrival and angle of arrival information is also delivered (in relation to (7), the angles are measured with respect to the inclination of the street and an additional delay must be included to express the distance traveled from the source to the wedge). The total average power can be estimated by the resulting geometric series

$$
\hat{P}_{\mathrm{est}}=\sum_{i} P_{i}=P_{\mathrm{SD}} \frac{1}{1-r_{\mathrm{opt}}} .
$$

The performance of the proposed approximation, compared to the exact calculation of all diffracted multiply reflected paths, is assessed in the following example. For wedge 1, shown in Fig. 3, we consider $n=1.5$ (90 ${ }^{\circ}$ interior angle), and $a$ and $k$ varying from 1 to 50 at unit steps. For each receiver's position, we specify $r_{d}$ by calculating $Y(0)$ and $Y(1)$, while $r_{R}(k)$ is calculated for several values of $k$. We also calculate $r_{\mathrm{opt}}^{\prime}=$ $\left(\hat{P}-P_{\mathrm{SD}}\right) / \hat{P}$, where $\hat{P}$ is the exact average power received at each position given in (8). The following statistics are gathered.

- the prediction error $e_{P}$ in $\mathrm{dB}$ defined as $e_{p}=$ $\left|\hat{P}(\mathrm{dBm})-\hat{P}_{\text {est }}(\mathrm{dBm})\right|$;

- the standard deviation $\sigma_{e p}$ of $e_{P}$;

- the error $e_{r}$, defined as $e_{r}=\left|r_{\mathrm{opt}}^{\prime}-r_{\mathrm{opt}}\right|$;

- the standard deviation $\sigma_{e r}$ of $e_{r}$.

We have found that $e_{p}>2 \mathrm{~dB}$ and $e_{r}>0.15$ only for $a k<6$ (inside the shadow region). Outside this small region, the average prediction error $\widehat{e_{p}}=0.4 \mathrm{~dB}, \sigma_{e p}=0.18 \mathrm{~dB}, \widehat{e_{r}}=0.027$ and $\sigma_{e r}=0.02$. Therefore, under the proposed scheme, very good accuracy is achieved practically everywhere, except of the transition region.

The procedure shown in this section can be directly repeated for higher order of diffraction (double diffraction etc.) by replacing $P_{W}$ in (8) with the incident power at the $n$th wedge.

\section{CONSIDERATION OF SIDE-STREETS}

In the analysis so far, we have shown that the $i$ th arriving ray from a main or a secondary source can be well approximated by a term in the form $P_{0} r_{\text {opt }}^{i}\left(r_{\mathrm{opt}}<1\right)$, where $i$ denotes the number of reflections that the ray has suffered in the street, where both the source and the receiver are located (referred to as receiver's street from now on). When side streets are considered between the receiver and the source, some rays never reach the receiver. By introducing an indicator function $I(i)$, this can be expressed as

$$
P_{i}=P_{0} r_{\mathrm{opt}}^{i} I(i)
$$

where

$$
I(i)= \begin{cases}1, & \text { if all } i \text { reflections occured } \\ 0, & \text { else. }\end{cases}
$$

We wish to associate with each order of reflection a suitable probability, describing whether this path exists. A reflection is to be included in the calculations if and only if the point of reflection lies on the surface of a surrounding building, as shown in Fig. 4. Assuming that we have no knowledge of the arrangement of the buildings around the receiver's street, the probability that a reflection actually occurred is given by

$$
p_{\text {ref }}=\frac{l_{b}}{l_{b}+l_{s}}
$$




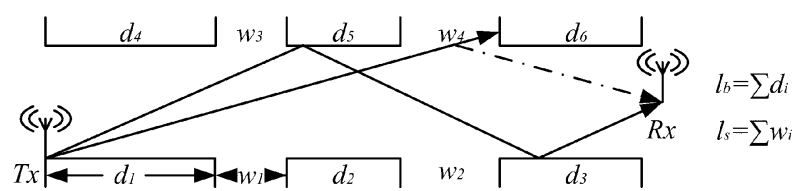

Fig. 4. Some paths never reach the receiver, when considering side streets.

where $l_{b}$ is the total length of the buildings' facades at the receiver's street and $l_{s}$ is the total width of the side streets. Both parameters can be easily specified for each street. Furthermore, we could assume that the occurrence of each successive reflection is independent from the previous one; the validity of which is strengthened by the fact that successive reflections occur at opposite sides of the receiver's street. In such a case the probability that $i$ reflections occurred is given by

$$
p_{\text {ref }}(i)=p_{\text {ref }}^{i}, \text { for } i \geq 0 .
$$

Now $I(i)$ equals 1 with probability $p_{\text {ref }}(i)$. Notice that $p_{\text {ref }}(0)=1$, since the direct path always exists. In such a case the average received power $\hat{P}_{\text {est }}$ at each position is given as

$$
\hat{P}_{\mathrm{est}}=\sum_{i} P_{0} r_{\mathrm{opt}}^{i} p_{\mathrm{ref}}^{i}=P_{0} \frac{1}{1-r_{e q}} \text {, with } r_{e q}=r_{\mathrm{opt}} p_{\mathrm{ref}}
$$

We have managed to associate a probability with each raypath that depends on the specific characteristics of the environment. For streets with sparsely arranged buildings, the multiply reflected components are not expected to contribute much in the total received power. Indeed, this is depicted in (13), where $l_{b}$ is expected to be small compared to $l_{s}$, and then in (15).

Equations (12) and (14) complement (7) and (10) to provide site-specific statistical outputs such as coverage probability, outage probability, statistics of fading, etc., taking into account the fact that part of the energy is guided into side streets, by assigning a suitable probability. Even more, by varying the parameter $p_{\text {ref }}$ from 1 to 0 , one can analyze extreme behaviors encountered in the urban canyon from perfect wave-guiding to negligible reflection-contribution, respectively, as will be shown in the following section.

\section{DisCUSSION-NuMERICAL EXAMPLES}

\section{A. Application of the Proposed Approximation in a Ray-Tracing Tool}

The implementation of the proposed propagation-prediction model can be accomplished by adopting the following eight steps. Altogether, as in [16] and [17], we need to determine the direct path (LOS or through diffraction) and the width of the street where the receiver is located at.

The first three steps are to be taken in a preprocessing phase, since they are associated only with the properties of the environment.

Step 1) Construction of the visibility relations between all wedges of the study area.

Step 2) Calculation and storage of $r_{\mathrm{opt}}(k)$ in (6) and $r_{R}(k)$ in (10) for each different material in the study area.
Step 3) Calculation and storage of $p_{\text {ref }}$ and the average width of each street in the study area.

When a transmitter is placed in the study area and the receivers' positions are specified, the following steps complete the process.

Step 4) Calculation of the wedges that are directly illuminated by the transmitter (completes the visibility tree of step 1).

\section{For each receiver:}

Step 5) Calculation of all sources (transmitter or wedges) that unobstructively illuminate it.

Step 6) From the visibility tree constructed in steps 1 and 4, all direct contributions (without reflections) from these sources are calculated and stored at decreasing received signal strength order.

Step 7) Calculation of $r_{d}$ in (10) only for the direct contributions that are within a user-defined range from the strongest one (10-15 dB is considered sufficient).

Step 8) Calculation of $\hat{P}_{\text {est }}$ in (15) [all necessary ratios are specified in steps (2), (3) and (7)].

Before execution no knowledge for the location of the receiver with respect to the shadow boundaries (SBs) is available. Therefore, UTD needs to be implemented in step 7, to avoid the singularities introduced at the SBs by the GTD. Within a transition region, around a SB, $r_{d}$ could be such that $r_{\mathrm{opt}}$, given in (10), could become greater than 1 . In such a case, $r_{\mathrm{opt}}$ is set to 0 ; yet, the direct contribution is accurately calculated through UTD and included in the calculations. An alternative approach that could be implemented is to control whether the receiver lies within the transition region and to calculate only the direct contribution if it does (a safe criterion is to control whether the argument of the Fresnel transition function of the UTD formulation is smaller than 10).

\section{B. Experimental Results}

We compare the estimations of the average power $P_{\text {est }}$ obtained by the proposed model with those acquired by implementing the analytical formulas presented in [17], $P_{2005}$, and with measurements carried out along the NLOS path $B-C$ of the typical urban configuration shown in Fig. 5 (Thessaloniki, Greece). A $2 \mathrm{GHz}$ carrier was transmitted at $27.5 \mathrm{dBm}$ by an antenna with $7.5 \mathrm{dBi}$ gain, manufactured by Huber-Suhner (model 1319.26.0024). The antenna was placed on a mast at $6 \mathrm{~m}$ height in front of a building at Svolou str. The receiving antenna was a $\lambda / 2$ dipole, fixed on top of a vehicle at $4 \mathrm{~m}$ height from the ground. The bandwidth of the measurement system is $100 \mathrm{kHz}$. The receiver moves from LOS to NLOS conditions along path $A-B-C$ for a distance of $R_{1}=220 \mathrm{~m}$ and $R_{2}=415 \mathrm{~m}$ respectively. The path is surrounded by eight story buildings. Therefore, the receiving and transmitting antennas can be considered well below rooftop level. The normalized $l_{b}(13)$ for Svolou str. 


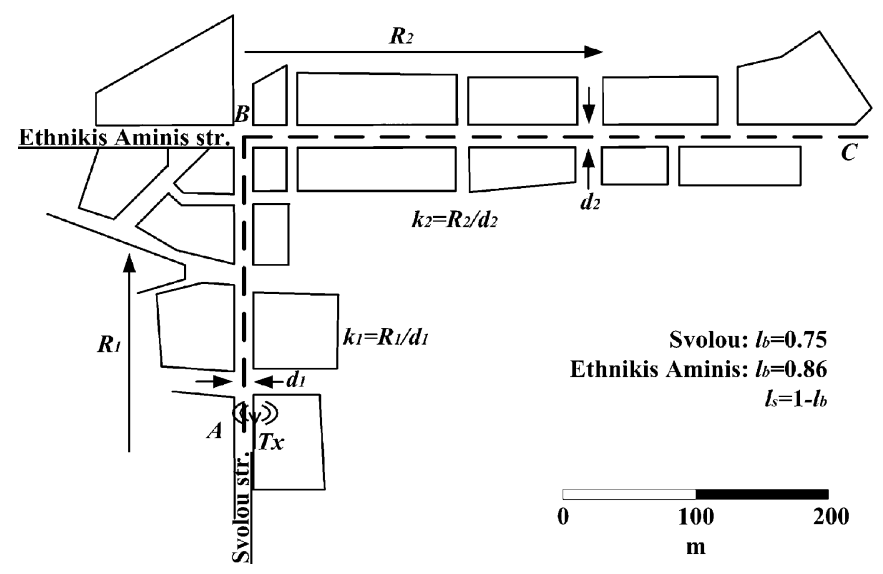

Fig. 5. Map of the simulation streets.

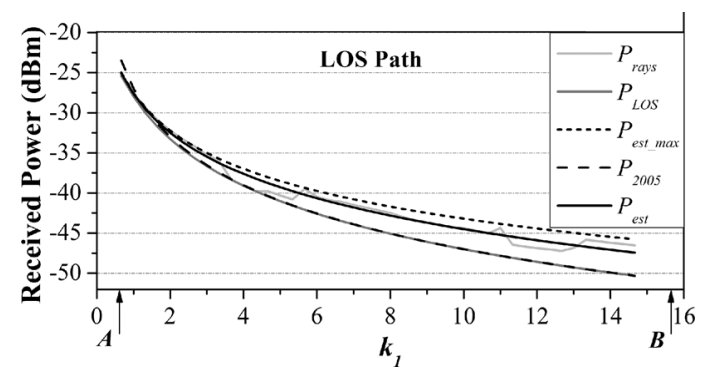

Fig. 6. Estimated received power for path $\mathrm{AB}$ (LOS), shown in Fig. 5.

and Ethnikis Aminis str. are 0.75 and 0.86 , respectively. For the facades, we have assumed $\varepsilon_{r}=6.05, \tan \delta=0.27$ [2].

For the part of the route where LOS conditions are satisfied $(A-B)$, we also calculate the direct only contribution $P_{\mathrm{LOS}}$, the estimated power $P_{\text {est_max }}$, as given by (6), ignoring the side streets and the average power $P_{\text {rays }}$ by calculating the contribution of the LOS ray and all multiply-reflected rays implementing a deterministic ray-tracing model. The results for this comparison are demonstrated in Fig. 6. The proposed approximation predicts satisfactorily $P_{\text {rays }}$. As expected, $P_{\text {LOS }}$ and $P_{\text {est_max }}$ represent the lower and the upper boundary of the variation of the average predicted power, respectively. The criterion set in (13)-(15) specifies adequately the variation of the estimated average received power with respect to the above two limits. In the geometry of Fig. $5, l_{b}$ is large; hence $P_{\text {est }}$ is closer to $P_{\text {est_max }}$. For a street with sparsely arranged buildings both $P_{\text {est }}$ and $P_{\text {rays }}$ would be closer to $P_{\mathrm{LOS}} . P_{2005}$ matches the $P_{\mathrm{LOS}}$ curve, because the authors in [17] attempt to specify and calculate the contribution of the single dominant path, which is the LOS ray in this case. As a consequence, at greater distances, where the multiply reflected rays become significant, $P_{2005}$ suffers from large errors. This property is also verified through measurements that are presented in Fig. 7.

The measurements along Ethnikis Aminis str. were conducted after midnight, in order to avoid temporary, yet strong contributions from elevated scatterers, such as buses, tracks etc., accepting a possible small error from not considering the contribution of the ground reflected ray. The received power was measured at $0.5 \mathrm{~s}$ time-intervals, resulting in distance-intervals of $1.65 \mathrm{~m}$. The measurements were repeated three times

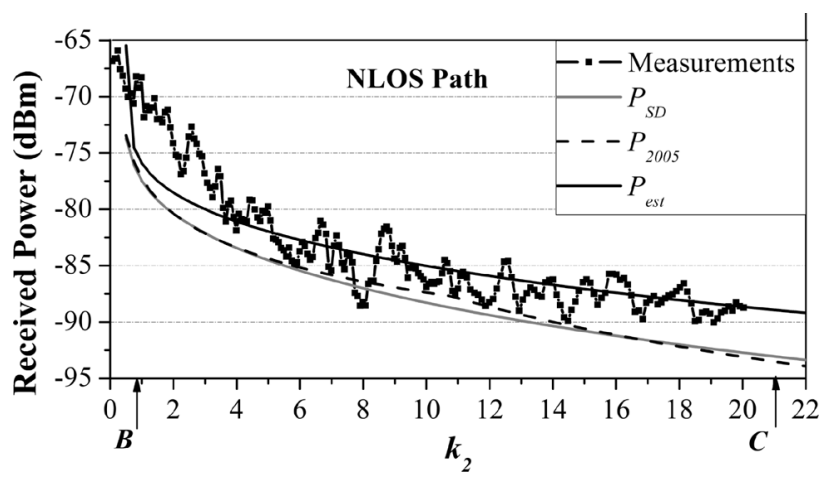

Fig. 7. Estimated received power versus measurements for path (BC), shown in Fig. 5.

along the same route, to account for temporal variations due to the small traffic that was encountered. In order to evaluate the average power, the following procedure was adopted. First, the average power (in Watts) of three consecutive samples along each route was calculated, and then the overall average for the three different repetitions of the process. Thus a total of nine samples were used for each result demonstrated in Fig. 7. The contribution of the singly diffracted ray $P_{\mathrm{SD}}$ is also plotted. The curves that represent $P_{\text {est_max }}$ and $P_{\text {rays }}$, which includes the contribution of all multiply reflected and diffracted-multiply-reflected rays by implementing a deterministic ray-tracing model [5], were not sketched in the graph, because their variation with respect to $P_{\text {est }}$ was similar to the one presented in the LOS case. In [17], the authors calculate the contribution of the strongest multiply reflected ray plus the singly diffracted one. For the calculations of $P_{2005}$ the reflection coefficient was considered equal to 0.5 . Since the crossroad is away from the transmitting antenna, $P_{2005}$ is almost equal to $P_{\mathrm{SD}}$, which means that the contribution of the multiply reflected ray is estimated to be smaller than that of the singly diffracted component [17]. After $50 \mathrm{~m}(k=5)$ from the crossroad, the proposed model agrees well with the measurements. The slope of $P_{\text {est }}$ seems to better approximate the measured samples than $P_{2005}$. This implies that at larger distances from the crossroad, the contribution of the diffracted-multiply-reflected rays should be included in the estimations. At smaller distances from the crossroad, the measured profile is stronger than the predicted one. The 1 st order multiply reflected rays that are not included in the calculations were expected to dominate in this small area.

\section{Prediction Time Analysis}

Compared to a deterministic ray-tracing model, significant time is saved by avoiding the exhaustive search of all multiply reflected rays. In the proposed model estimations are delivered as soon as all sources (real and secondary) that illuminate each receiver are specified (steps 4 and 5 of Section V-A). Let us assume an area with $M$ secondary sources (wedges). In step 4, $M$ rays must be considered from the source to each of the wedges in the study area, in order to specify the wedges that are directly illuminated by the transmitting antenna. Hence, the time to perform this test is $\mathrm{O}(M)$. The same test needs to be repeated for each receiver in the study area in step 5. Assuming 


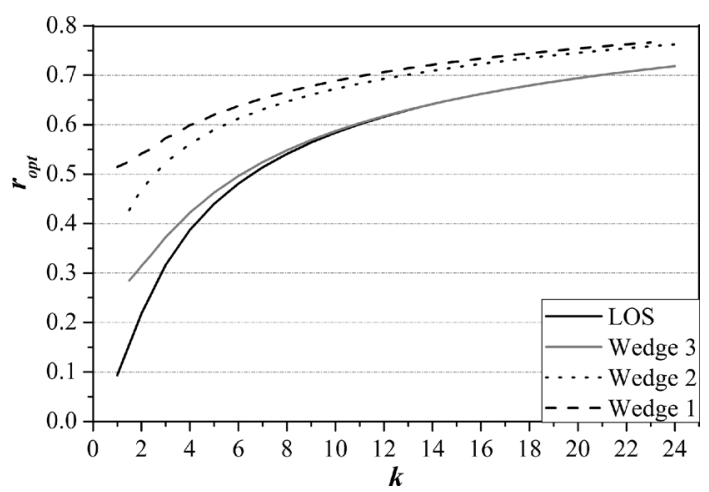

Fig. 8. Variation of $r_{\mathrm{opt}}$ for the scenario of Fig. 5 .

$N$ receivers, the worst case running time to complete steps 4 and 5 is $\mathrm{O}(N M)$.

In a deterministic ray-launching model, rays are initiated from each source (real and secondary) and for each ray the intersection with the closest obstacle must be specified. Assuming that the aforementioned $M$ wedges are sections of buildings, a total of $M$ walls exist in the area of interest. In such a case, the worst-case running time to specify the intersection of a ray with the closest obstacle is $\mathrm{O}(M)$. We assume that the contribution of each ray becomes negligible after it has suffered $n$ reflections, and a total of $K$ rays are launched from each of the $M+1$ real and secondary sources. The worst case running time to specify all multiply reflected rays is $\mathrm{O}\left(M^{2} K n\right)$.

The typical coverage area of an antenna located well below rooftop level does not exceed $500 \mathrm{~m} \times 500 \mathrm{~m}$. In such a typical Mediterranean urban area, 60 building-blocks (about 250 wedges) are located. Assuming that the receivers are arranged every $5 \mathrm{~m}$ outside the buildings results in approximately $N \approx$ 1000 receivers. In a deterministic ray-launching model, typical values for $n$ and $K$ are 7 and 720, respectively. In such a case, the worst case running time of the ray-launching approach is 1260 times greater than that of the proposed model. In the above example, the time to specify the sources in the proposed model was 12.4 seconds in a laptop operating under Windows XP with a Pentium M 1.5 GHz processor and 512 MB RAM. The corresponding time for a deterministic model could be as high as 4 hours (corresponding to the above worst-case analysis).

\section{Variation of $r_{\mathrm{opt}}$}

$r_{\text {opt }}$ represents a measure of the contribution of secondary ray-paths at each position. A small value of $r_{\mathrm{opt}}$ implies that the direct path dominates, whereas a larger value means that secondary terms contribute significantly to the receiver.

In Fig. 8, $r_{\text {opt }}$ is plotted in connection with $k$, for the scenario of Fig. 5. We have removed the influence of side streets [introduced through (15)], in order to compare the variation of the LOS ratio $r_{\mathrm{LOS}}$ given by (6) with that due to the wedges, given by (11). Notice that the ratio due to wedge 4 is not plotted, since the contribution of the latter was smaller than $20 \mathrm{~dB}$ than that of wedge 3 which dominates.

In all four cases, the ratio grows as the distance from the source increases. This is due to the fact that at greater distances,

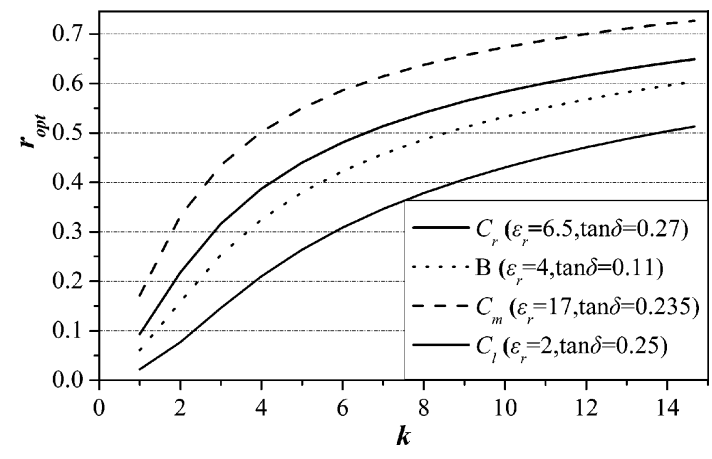

Fig. 9. Variation of $r_{\mathrm{opt}}$ for materials, typically encountered in the "urban scene."

reflection occurs at a shallow angle and the magnitude of the corresponding coefficient grows. Therefore, more terms contribute significantly to the total received field, as mirrored at greater values of $r_{\mathrm{opt}}$. When the influence of side streets is included, all samples in Fig. 8, have to be reduced by the appropriate factor, depending on $l_{b}$.

The variation of $r_{\mathrm{opt}}$ for a LOS case, as specified by (6), for the following typical materials is shown in Fig. 9: a) reinforced concrete $\left(C_{r}\right)$ [2], b) brick $(B)$ [25], c) concrete with $30 \%$ moisture $\left(C_{m}\right)$ [26], and d) lightweight concrete $\left(C_{l}\right)$ [27]. The greater the magnitude of the reflection coefficient is, the greater the value of $r_{\text {opt }}$ for the same $k$.

Notice that in the graphs of Figs. 8 and 9, $r_{\text {opt }}$ is plotted for increasing $k$. Thus for wider streets, $r_{\mathrm{opt}}$ equals a specific value at a greater distance $d_{w}$ compared to that for a narrow street $d_{n}$, given by $d_{w}=d_{n}\left(w_{w} / w_{n}\right)$, where $w_{w}$ and $w_{n}$ are the corresponding widths of the two streets.

As shown in [20], if foliage exists along a street, additional attenuation that depends on the distance traveled through vegetation must be computed for all components. Due to the larger path through vegetation, the contribution of the multiply reflected rays compared to the direct ones becomes negligible. Hence, $r_{\mathrm{opt}}$ approximates 0 , and the average expected power is given by the direct-only components $\left(P_{\mathrm{SD}}\right.$ and $\left.P_{\mathrm{LOS}}\right)$.

Therefore, the parameter $r_{\mathrm{opt}}$, introduced in this paper, carries important information on the contribution of secondary raypaths associated with each source, taking into account both the geometry and the EM properties of the materials of the specific environment.

\section{E. Range of Application}

In the analysis so far, we have assumed that the receiver is at the center of the street that was studied. Similar behavior was also recorded when the receiver was considered close to each of the two sides of the street, even though $r_{\mathrm{opt}}$ in (6) and (10) was calculated for a position at the center of the street. High accuracy was also delivered for directional antennas. In addition, similar results were verified for a transmitter placed at a different height compared with the receiver ( $6 \mathrm{~m}$ for a receiver at $1.8 \mathrm{~m})$. For the cases under examination, we do not need a three dimensional approach as long as both the transmitter and the receiver are located within the canyon, regardless of its inclination with respect to the horizon. 
A special case that deserves attention is when the receiver is at a square. In such a case, $k$ is expected to be small. Thus, diffraction could occur in the transition region. As stated previously in Section V-A, by implementing the proposed approximation, only the direct contribution would be calculated. As shown in Figs. 6 and 7, for small values of $k$, the direct contribution dominates ( $r_{\mathrm{opt}}$ is very small and curves $P_{\mathrm{SD}}$ and $P_{\text {est_max }}$ coincide). Hence, also for this special case high accuracy is expected.

\section{CONCLUSION}

In this paper, we put forward a new semi-deterministic propagation prediction model. The idea is to identify all sources and associate a suitable geometric progression with each one that accurately describes the contribution of secondary terms. Under this approach, estimation is delivered as soon as the main contribution and the ratio have been specified. The ratio of each progression reflects the significance of secondary ray-paths at each position. It carries important information on the geometry and the EM properties of the materials inside a "street-canyon," and can be treated as a new measure of the scattering behavior of each environment.

The presented model is not limited in path-loss only prediction. Instead, detailed information on the magnitude, the time of arrival and the angle of arrival of all important components is acquired in a short running-time. Therefore, it is ideal for the wideband characterization of the environment, which is important for all modern communication-systems.

High accuracy was achieved for a typical urban configuration. The proposed concept could also be extended for an antenna placed above rooftop level. In a similar reasoning, the main contribution would be the over-rooftop multiply diffracted component and a suitable progression could describe the multiply reflected components at the vicinity of the receiver in the "canyon."

\section{APPENDIX}

We show that away from the 2 SBs the derivative of $\Lambda(i)$ with respect to $i$ approaches 0 . For simplicity, for the heuristic diffraction coefficient [8], we will assume that the reflection-coefficients associated with the two facets of the wedge are equal and real. Outside the transition region, the Fresnel transition function equals one. So we have for $D(i)$

$|D(i)|^{2}=G\left(\frac{1}{\cos \left(\frac{\phi-\phi^{\prime}(i)}{n}\right)-\cos \frac{\pi}{n}}+\Gamma \frac{1}{\cos \left(\frac{\phi+\phi^{\prime}(i)}{n}\right)-\cos \frac{\pi}{n}}\right)^{2}$

where $G$ is a common term for all $i$ [24] and $n$ is associated with the interior wedge angle (ranges from 1 to 2 ). For $\Gamma= \pm 1$, we get the original Keller's solution [24] for the Neumann and the Dirichlet boundary conditions respectively. The distance depending term will be written $A(i)$ and let $F(i)=|D(i)|^{2}$. After some simplifications, the derivative is written as

$$
\begin{aligned}
\frac{d \Lambda(i)}{d i} & =\frac{\left[\frac{d F(i+1)}{d i} A(i+1)+F(i+1) \frac{d A(i+1)}{d i}\right] F(i) A(i)}{(F(i) A(i))^{2}} \\
& -\frac{\left[\frac{d F(i)}{d i} A(i)+F(i) \frac{d A(i)}{d i}\right] F(i+1) A(i+1)}{(F(i) A(i))^{2}} .
\end{aligned}
$$

We can write

$$
\begin{aligned}
\left|\frac{d \Lambda(i)}{d i}\right| \leq & \left|\frac{\frac{d F(i+1)}{d i} A(i+1) F(i) A(i)}{(F(i) A(i))^{2}}\right| \\
& +\left|\frac{F(i+1) \frac{d A(i+1)}{d i} F(i) A(i)}{(F(i) A(i))^{2}}\right|+ \\
& +\left|\frac{\frac{d F(i)}{d i} A(i) F(i+1) A(i+1)}{(F(i) A(i))^{2}}\right| \\
& +\left|\frac{F(i) \frac{d A(i)}{d i} F(i+1) A(i+1)}{(F(i) A(i))^{2}}\right| .
\end{aligned}
$$

Let us consider the ratio

$$
\begin{aligned}
& \frac{\frac{d A(i)}{d i}}{A(i)}=\cdots=\frac{\frac{(2 i+1)}{2}}{k^{2}+\left(\frac{(2 i+1)}{2}\right)^{2}} \\
& +\frac{\frac{(2 i+1)}{2}}{\left[k^{2}+\left(\frac{(2 i+1)}{2}\right)^{2}\right]^{1 / 2}\left[a+\left[k^{2}+\left(\frac{(2 i+1)}{2}\right)^{2}\right]^{1 / 2}\right]} \ll 1 .
\end{aligned}
$$

The denominator of the 2 nd term is greater than that of the 1 st. It is obvious that both terms are much smaller than 1 for greater values of $k$ and $i \geq 0$. Similarly, by considering the ratio $(d F(i) / d i) / F(i)$ for the four cases of wedges, shown in Fig. 3 and assuming for the typically encountered geometries that $d \varphi=d \varphi^{\prime}(i) \approx 0$, we have

$$
\left|\frac{\frac{d F(i)}{d i}}{F(i)}\right|=|H(i)||Q(i)|, \text { where } H(i)=\frac{8 k}{n\left(4 k^{2}+(2 i+1)^{2}\right)}
$$

and

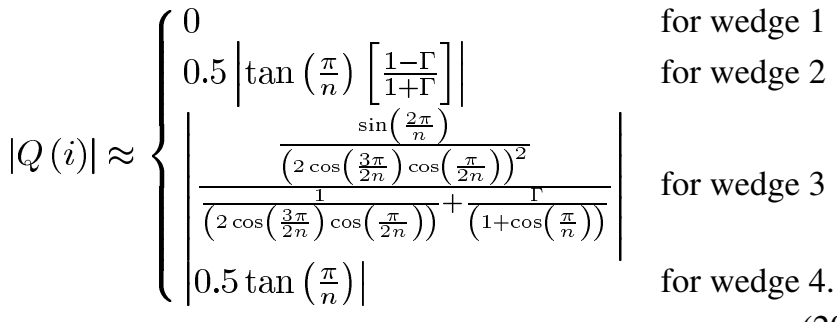

For wedges 2 and 3, if the value of $\Gamma$ is such that the denominator of $Q(i)$ is zero, the corresponding diffracted field given by (16) is also zero and hence such terms are not expected to contribute in the proposed approximation. The term $|0.5 \tan (\pi / n)|$ remains smaller than 5 for $n \leq 1.88$. For greater values of $n$, diffraction occurs in the transition region for wedges 2 and 4 . Similarly, for wedge 3, when diffraction occurs outside the SB ( $n \geq 1.2$ ), the corresponding term is very small. For greater values of $k$ and $i \geq 0,|H(i)| \ll 1$. Therefore, provided that the receiver lies outside the SBs of the corresponding diffraction and the diffraction coefficient term is different than zero, we have

$$
\left|\frac{\frac{d F(i)}{d i}}{F(i)}\right| \ll 1
$$


By inspecting Fig. 3, we can easily deduce that for larger $k$, the term $A(i+1)$ is comparable with $A(i)$ due to the small distance difference and $F(i+1)$ with $F(i)$, due to the small angular separation. Similarly to (19) and (21), we can show that $(d F(i+1) / d i) / F(i) \ll 1$ and $(d A(i+1) / d i) / A(i) \ll 1$. Hence

$$
\left|\frac{d \Lambda(i)}{d i}\right| \ll 1 \Leftrightarrow\left|\frac{d \Lambda(i)}{d i}\right| \approx 0
$$

Had we considered the subtraction shown in (17), the derivative would have been proven even smaller. In fact for $n=1.5, \Gamma=$ $1.0, k=10, a=5$, the derivative ranges between $10^{-2}$ to $10^{-5}$ for all four geometries and $i<10$.

\section{REFERENCES}

[1] S. R. Saunders, Antennas and Propagation for Wireless Communication Systems. New York: Wiley, 1999.

[2] M. F. Catedra and J. Perez-Arriaga, Cell Planning for Wireless Communications. Boston-London: Artech House, 1999.

[3] T. K. Sarkar, Z. Ji, K. Kim, A. Medour, and M. Salazar-Palma, "A survey of various propagation models for mobile communications," IEEE Antennas Propag. Mag., vol. 45, no. 3, pp. 51-82, Jun. 2003.

[4] D. Har, H. H. Xia, and H. L. Bertoni, "Path-loss prediction model for microcells," IEEE Trans. Veh. Technol., vol. 48, pp. 1453-1462, Sep. 1999.

[5] G. E. Athanasiadou and A. R. Nix, "A novel 3-D indoor ray-tracing propagation model: The path generator and evaluation of narrow-band and wide-band predictions," IEEE Trans. Veh. Technol., vol. 49, pp. 1152-1168, Jul. 2000.

[6] A. G. Dimitriou and G. D. Sergiadis, "A novel, shooting and bouncing rays technique for propagation prediction, based on a varying-step calculation procedure," in Proc. 5th Mediterranean Microwave Symp., Athens, Sep. 2005, pp. 34-39.

[7] R. G. Kouyoumjian and P. H. Pathak, "A uniform geometrical theory of diffraction for an edge in a perfectly conducting surface," Proc. IEEE, vol. 62, pp. 1448-1461, Nov. 1974

[8] R. J. Luebbers, "Finite conductivity uniform GTD versus knife edge diffraction in prediction of propagation path loss," IEEE Trans. Antennas Propag., vol. 32, pp. 70-76, Jan. 1984.

[9] G. E. Athanasiadou and A. R. Nix, "Investigation into the sensitivity of the power predictions of a microcellular ray tracing propagation model," IEEE Trans. Veh. Technol., vol. 49, pp. 1140-1151, Jul. 2000.

[10] Z. Chen, H. L. Bertoni, and A. Delis, "Progressive and approximate techniques in ray-tracing-based radio wave propagation prediction models," IEEE Trans. Antennas Propag., vol. 52, pp. 240-251, Jan. 2004.

[11] A. G. Dimitriou and G. D. Sergiadis, "Managing topological prioritization in ray-tracing based progressive propagation-prediction modeling," IEEE Trans. Antennas Propag., vol. 54, pp. 3042-3046, Oct. 2006.

[12] Digital mobile radio toward future generation systems: Final Report European Commission, Brussels, 1999, European COST action 231.

[13] F. Fuschini, V. D. Esposti, and G. Falciasecca, "A statistical model for over rooftop propagation," IEEE Trans. Antennas Propag., vol. 52, pp. 230-239, Jan. 2004.

[14] H. Xia, "A simplified analytical model for predicting path loss in urban and suburban environments," IEEE Trans. Veh. Technol., vol. 46, pp. 1040-1046, Nov. 1997

[15] N. C. Gonçalves and L. M. Correia, "A propagation model for urban microcellular systems at the UHF band," IEEE Trans. Veh. Technol., vol. 49, pp. 1294-1302, Jul. 2000.

[16] W. Zhang and N. Moayeri, "Closed-form expressions for the prediction of microcellular mobile radio propagation in urban environments," IEEE Trans. Antennas Propag., vol. 51, pp. 952-956, May 2003.

[17] Q. Sun, S. Y. Tan, and K. C. Teh, "Analytical formulae for path loss prediction in urban street grid microcellular environments," IEEE Trans. Veh. Technol., vol. 54, pp. 1251-1258, Jul. 2005.
[18] J. Lee and H. L. Bertoni, "Coupling at cross, T, and L junctions in tunnels and urban street canyons," IEEE Trans. Antennas Propag., vol. 51, pp. 926-935, May 2003.

[19] J. Laurila, K. Kalliola, M. Toeltsch, K. Hugl, P. Vainikainen, and E. Bonek, "Wideband 3-D characterization of mobile radio channels in urban environment," IEEE Trans. Antennas Propag., vol. 50, pp. 233-243, Feb. 2002

[20] A. G. Dimitriou and G. D. Sergiadis, "Architectural features and urban propagation," IEEE Trans. Antennas Propag., vol. 54, pp. 774-784, Mar. 2006

[21] V. Erceg, A. J. Rustako, and R. S. Roman, "Diffraction around corners and its effects on the microcell coverage area in urban and suburban environments at $900 \mathrm{MHz}, 2 \mathrm{GHz}$, and $4 \mathrm{GHz}$," IEEE Trans. Veh. Technol., vol. 43, pp. 762-766, Aug. 1994.

[22] K. Rizk, J. F. Wagen, and F. Gardiol, "Two-dimensional ray-tracing modeling for propagation prediction in microcellular environments," IEEE Trans. Veh. Technol., vol. 46, pp. 508-518, May 1997.

[23] L. Piazzi and H. L. Bertoni, "Achievable accuracy of site-specific path-loss predictions in residential environments," IEEE Trans. Veh. Technol., vol. 48, pp. 922-930, May 1999.

[24] J. B. Keller, "Geometrical theory of diffraction," J. Opt. Soc. Amer., vol. 52, no. 2, pp. 116-130, Feb. 1962.

[25] D. Peña, R. Feick, H. D. Hristov, and W. Grote, "Measurement and modeling of propagation losses in brick and concrete walls for the 900-MHz band," IEEE Antennas Propag. Mag., vol. 51, no. 1, pp. 31-39, Jan. 2003

[26] J. B. Hasted and M. A. Shah, "Microwave absorption by water in building materials," British J. App. Phys., vol. 15, pp. 825-836, 1964.

[27] Recommendation ITU-R P. 1238-4 ITU, 2005.

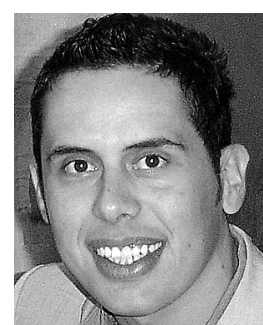

Antonis G. Dimitriou ( $\mathrm{S}^{\prime} 01$ ) was born in Ierapetra, Greece, in 1977. He received the Diploma and the $\mathrm{Ph} . \mathrm{D}$ degrees in electrical and computer engineering from the Aristotle University of Thessaloniki, Greece, in 2001 and 2006 respectively.

Since 2001, he has been a Teaching and Research Assistant in the Department of Electrical and Computer Engineering of the Aristotle University of Thessaloniki, where he has participated in numerous research activities, including the design of a GSM cellular network inside the Olympic Stadium for the 2004 Olympic Games. His current interests are in the areas of antennas, propagation modeling, using high frequency methods in electromagnetics and planning.

Dr. Dimitriou is a member of the technical chamber of Greece.

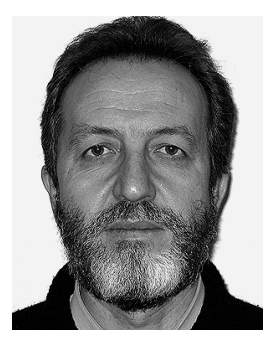

George D. Sergiadis (M'88) was born in Thessaloniki, Greece, in 1955. He received the Diploma degree in electrical engineering from the Aristotle University of Thessaloniki, Thessaloniki, Greece, in 1978, and the Ph.D. degree from "Ecole Nationale Supérieure des Télécommunications," Paris, France, in 1982.

He worked with Thomson CsF, in France, until 1985, participating in the development of the French Magnetic Resonance Scanner. Since 1985, he has been with the Aristotle University of Thessaloniki, Greece, teaching telecommunications and biomedical engineering, where he is currently an Associate Professor. For three years he also served as the Director of the Telecommunications Department. He has developed the Hellenic TTS engine "Esopos," and designed the mobile communications for the Athens Olympic Games in 2004. His current research interests include fuzzy image processing and wireless communications. During the academic year 2004-2005 he was a Visiting Researcher at Media Lab, MIT, Cambridge, MA.

Dr. Sergiadis is the president of ELEVIT, the Hellenic society for Biomedical Engineering, and is a member of the Technical Chamber of Greece (TEE), ATLAS, SMRM, ESMRM, EMBS, and SCIP. 